\title{
Research on the Gait Planning of Artificial Leg Based on Central Pattern Generator
}

\author{
Hualong Xie ${ }^{1, \text { a }}$, Deming Zhao ${ }^{1, b}$, Zheng Jia ${ }^{1, c}$, Yuying Yang ${ }^{1, d}$ \\ ${ }^{1}$ Department of Mechanical Engineering and Automation, Northeastern University, Shenyang, \\ 110819, China \\ aemail: hlxie@mail.neu.edu.cn, bemail:neudeming@163.com \\ cemail: jiazhenghi@126.com, ${ }^{\mathrm{d}}$ email: yyyingneu@163.com
}

Keywords: Central Pattern Generator (CPG); Artificial Leg; Gait Planning; Genetic Algorithm

\begin{abstract}
To achieve the artificial leg of biped robot with heterogeneous legs (BRHL) humanoid walking, the gait planning of artificial leg was researched in this paper based on central pattern generator (CPG). Firstly, the hopf oscillator was analyzed and applied to build the CPG network. Then, the parameters of CPG network were optimized by genetic algorithm and the gait curves of CPG network planning were obtained by MATLAB simulation. Finally, based on ADAMS, the walking simulation of BRHL was carried out and the rationality of the gait planning of artificial leg by CPG network was verified.
\end{abstract}

\section{Introduction}

It is necessary to carry out a lot of performance testing in the process of research and development of intelligent prostheses. Due to the heavy workload of the experimental test and the requirement of good repeatability, it is not realistic for the disabled to carry on the experiment. To provide an ideal experimental platform for testing the performance of intelligent prosthetic, our research group proposed a biped robot with heterogeneous legs (BRHL), as shown in Figure 1. One leg of the robot is named artificial leg, which is used to replace the disabled person's healthy leg. The other leg is named bionic leg, which is used to imitate the intelligent prostheses worn by the disabled [1]. Artificial leg as an important part of BRHL, its main function is to imitate the normal gait of the disabled person's healthy leg and provide the bionic leg with gait target trajectory. Therefore, gait planning of the artificial leg has a very important significance for the study of the BRHL.

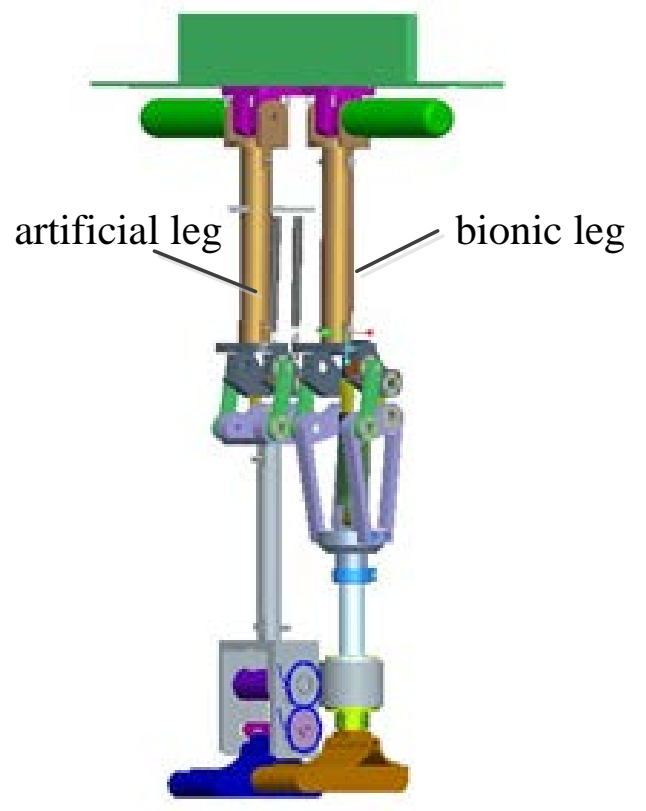

Fig.1. Virtual prototype model of BRHL 
The study of modern nervous system has proved that the rhythm movement of walking, breathing and swimming of the higher animals are controlled by the central pattern generator (CPG) [2]. CPG is a local oscillation network formed by neurons, which achieve self-excitation oscillation through mutual inhibition between neurons, then generate single or multiple cycle signals, which have the characteristics of stability and phase interlocking relationship to control the rhythmic movement of the body or body parts [3]. According to the biological characteristics of CPG, many scholars have attempted to use the CPG control mechanism to carry on the gait planning of the robot and some achievements have been obtained. In 1997, S.T.Ve-nkataraman used CPG to realize the gait generation and the control of six-leg insect robot, but the realization of the movement was relatively simple [4]. In 2000, Kimura used CPG to realize the control of four-leg robot, through the visual sensor information to generate nerve reflex and control CPG neurons "flexor" and "extensor" action [5] [6]. In 2005, Long Wang applied four Matsuoka oscillator model to establish the CPG control network and successfully realized the control of the bionic robot fish [7]. In view of the superiority of CPG to control the rhythmic movement, this paper uses 6 hopf nonlinear oscillators to build a CPG network for the gait planning of BRHL's artificial leg, so that the artificial leg's gait can be more human nature under the premise of satisfying stability.

\section{Mathematical Model Analysis of Hopf Oscillator}

At present, the hopf oscillator, matsuoka oscillator and rayleigh oscillator are widely used among the nonlinear oscillator models [8]. In view of the hopf oscillator model is relatively simple, the parameters are less and easy to optimize, this paper selects the hopf oscillator to build CPG network.

The mathematical expression of the hopf oscillator is as follows:

$$
\begin{aligned}
& \frac{d x}{d t}=\left(\mu-\gamma^{2}\right) * x+\omega * y \\
& \frac{d y}{d t}=\left(\mu-\gamma^{2}\right) * y-\omega * x
\end{aligned}
$$

In the type: $\gamma=\sqrt{x^{2}+y^{2}}, \mu>0 ; x$ is the oscillator output; $\mu$ is the amplitude of oscillator; $\omega$ is the frequency of the oscillator.

Two hopf oscillators can generate two different connection ways: one is named one-way connection, which means one of the two oscillators in the connection acts on the other; the other is named two-way connection, which refers to the interaction between the two oscillators.

One-way connection of the two oscillators mathematical expressions are as follows:

$$
\begin{aligned}
& \frac{d x_{1}}{d t}=\left(\mu_{1}-\gamma_{1}^{2}\right) * x_{1}+\omega * y_{1} \\
& \frac{d y_{1}}{d t}=\left(\mu_{1}-\gamma_{1}^{2}\right) * y_{1}-\omega * x_{1} \\
& \frac{d x_{2}}{d t}=\left(\mu_{2}-\gamma_{2}^{2}\right) * x_{2}+\omega * y_{2}+\varepsilon_{2} * x_{1} \\
& \frac{d y_{2}}{d t}=\left(\mu_{2}-\gamma_{2}^{2}\right) * y_{2}-\omega * x_{2}
\end{aligned}
$$

In the type: $\gamma_{i}=\sqrt{x_{i}^{2}+y_{i}^{2}} ; \quad \varepsilon_{2}$ is the connection weights; $x_{1}$ and $x_{2}$ are the outputs of the oscillator 1 and oscillator 2 .

After two oscillators are connected in one-way, the amplitude will be determined by $\mu_{i}$ and $\varepsilon_{2}$, this is because the two oscillators that have same frequency cause resonance. In addition, the phase difference of the oscillator output can be changed by changing the value of $\varepsilon_{2}$. If $\varepsilon_{2}>0$, the outputs of the two oscillators are in the same phase. If $\varepsilon_{2}<0$, the outputs of the two oscillators are 
in the opposite phase. The outputs of the oscillators are shown in Figure 2 and 3.

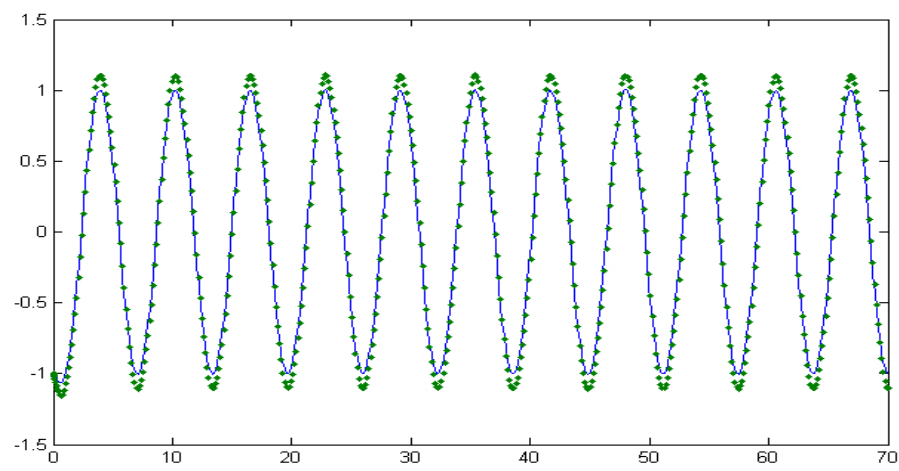

Fig.2. Two oscillators of unidirectional connection with the same phase output

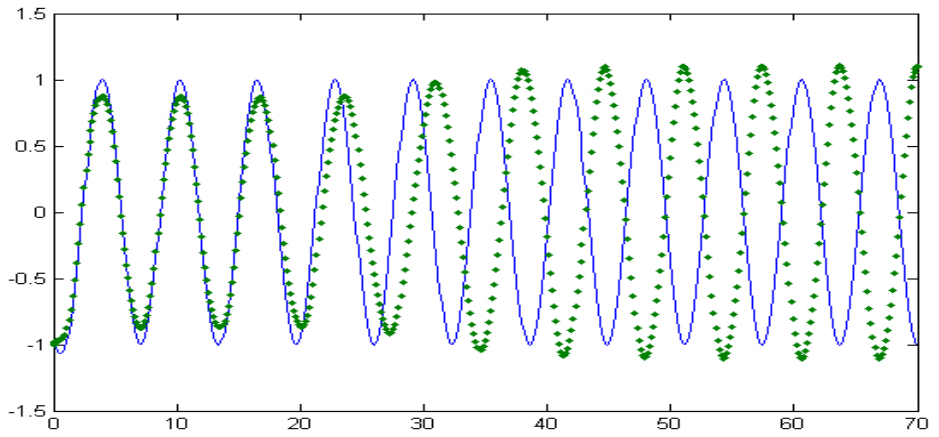

Fig.3. Two oscillators of unidirectional connection with the opposite phase output

By analyzing the hopf oscillator, it is known that a stable periodic oscillation signal can be produced by hopf oscillator, the amplitude and period of the output signal can be controlled by adjusting the parameters of the oscillator.

\section{CPG Network Build}

For BRHL, the structure of the bionic leg is regarded as the artificial leg, thus this paper completes the task of artificial leg gait planning by controlling the 6 joints of both legs. According to the movement of each joint when walking, six joints are numbered as the sequence of left hip joint, left knee joint, left ankle joint, right hip joint, right knee joint and right ankle joint, the phase sequence of the oscillators of hip joint and knee joint should be kept 1, 2, 4, 5, while 3 and 1, 6 and 4 should be in the same phase. CPG network is shown in Figure 4, the solid line represents the incentive and the dotted line represents inhibition.

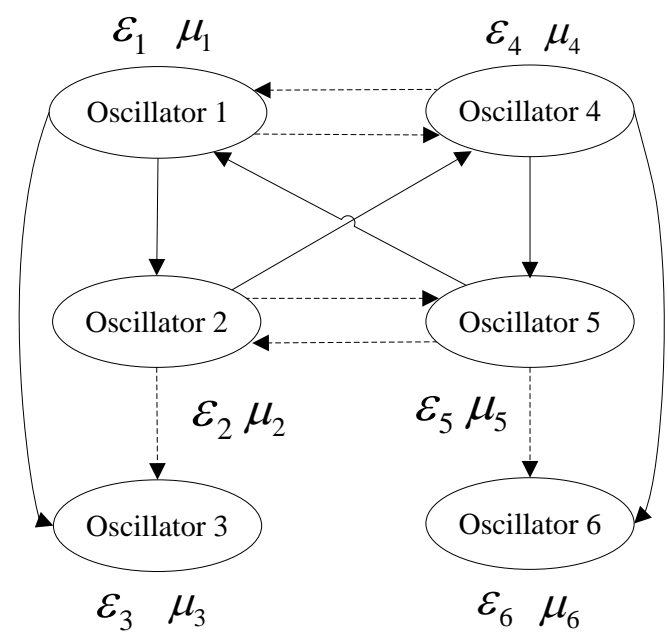

Fig.4. CPG network 


\section{CPG Parameters Optimization Based on the Genetic Algorithm}

Genetic algorithm (GA) is a kind of intelligent algorithm, which simulates the genetic and evolution mechanism of biology in nature to search for the optimal solution [9]. Given the powerful ability of optimization, to satisfy the needs of maximum stability and minimum energy consumption of the artificial leg gait, the genetic algorithm is adopted to optimize the parameters of CPG network in this paper. GA parameters are as shown in table1.

Table.1. Genetic algorithm parameters setting

\begin{tabular}{cc}
\hline name & Parameter setting \\
\hline Population size & 30 \\
The number of elite & 2 \\
Selective type & roulette wheel selection \\
Crossover probability & 0.6 \\
Cross type & Two-point crossover \\
The number of generation & 500 \\
Mutation probability & 0.1 \\
Mutation type & Uniform variation \\
\hline
\end{tabular}

In the genetic algorithm, the fitness function, also called evaluation function, the design of the fitness function is a key of the whole genetic algorithm. In this paper, fitness function is mainly used to evaluate the quality of artificial leg gait, so this paper chooses Zero Moment Point (ZMP) to evaluate it and introduces the walking stability margin $D_{s}$, namely minimum distance between ZMP point and the feet support plane boundary. When $D_{s}$ increasing, the walking stability will be higher. In order to ensure BRHL keep stable, ZMP should always stay in the feet support area and the robot support foot diagram is shown in Figure 5. The movement direction of the robot is in the $\mathrm{X}$-axis, so this paper will consider the change of the robot ZMP in X-axis direction only.

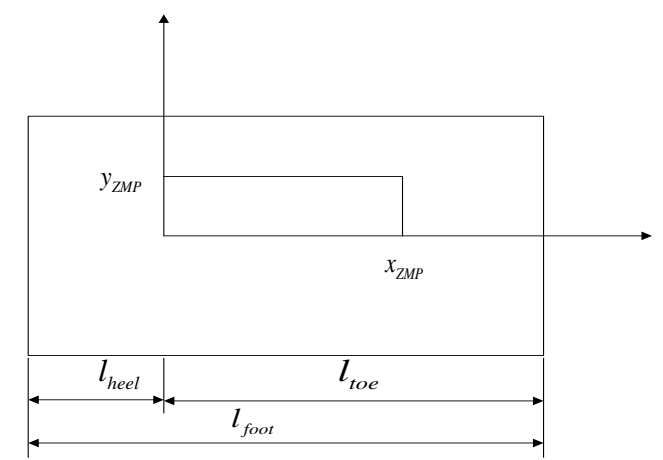

Fig.5. Schematic diagram of the feet support area

In the coordinate system, the origin is set as the ankle's subpoint in the feet supporting plane, $l_{\text {heel }}$ is the length of heel, $l_{\text {toe }}$ is the length of forefoot. In order to ensure that BRHL will not rotate in X-axis during walking, the ZMP should satisfy the following relationship:

$$
-l_{\text {heel }} \leq x_{\text {ZMP }} \leq l_{\text {toe }}
$$

The ZMP point on the forward way of robot walking can be obtained by D'Alembert's principle and the computation formula is shown as follows [10]:

$$
x_{\text {ZMP }}=\frac{\sum_{i=0}^{n} m_{i}\left(\ddot{z}_{i}+g_{z}\right) x_{i}-\sum_{i=0}^{n} m_{i}\left(\ddot{x}_{i}+g_{x}\right) z_{i}}{\sum_{i=0}^{n} m_{i}\left(\ddot{z}_{i}+g_{z}\right)}
$$

In the type: $m_{i}$ is the quality of linkage $i,\left(g_{x}, g_{z}\right)$ is the gravity acceleration, $\left(x_{i}, z_{i}\right)$ is the mass center of linkage $i$ in the XZ plane, which is obtained from forward kinematics equation. During BRHL walking, if the ZMP point can always be in the feet supporting plane, the stability of walking will be guaranteed. 
In view of the individual $\tilde{X}$ in the group, the objective function is shown as follows:

$$
f(\tilde{x})=D_{s}=\min \left\{\left|x_{Z M P}-l_{\text {toe }}\right|,\left|l_{\text {heel }}-x_{\text {ZMP }}\right|\right\}
$$

In this paper, the objective function is the minimum distance between the ZMP and the boundary valve of stable region, so the fitness function is shown as follows:

$$
\operatorname{Fit}(f(x))=\frac{1}{1+c+f(x)}
$$

Through genetic algorithm adjusting, the results are as shown in table 2 .

Table.2.The optimized CPG network parameters

\begin{tabular}{cccc}
\hline parameter & amplitude $\mu$ & frequency $\omega$ (rad/s) & weight $\varepsilon$ \\
\hline hip joint & 1.214 & 2.010 & 1.403 \\
knee joint & 1.051 & 2.010 & -1.579 \\
ankle joint & 0.890 & 2.010 & 1.065 \\
\hline
\end{tabular}

Taking the parameters into the CPG network which is built in the previous section, the gait planning results of CPG network are obtained by MATLAB simulation. The outputs of the oscillators are as shown in Figure 6.

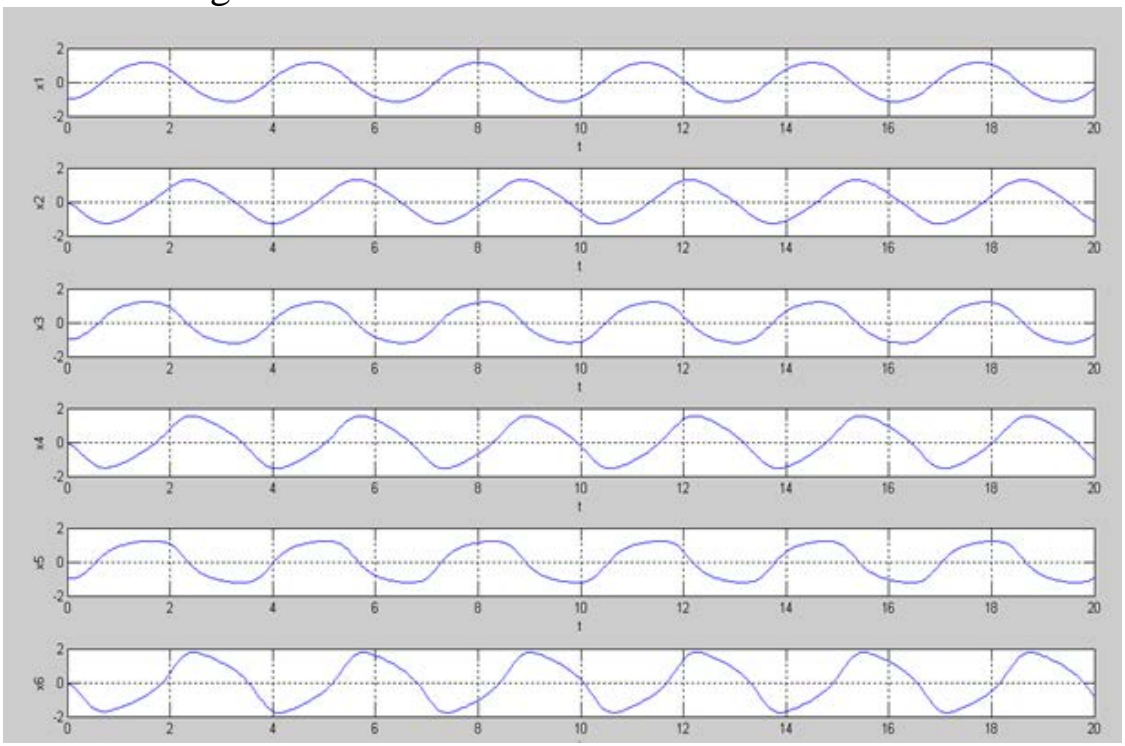

Fig.6. Waveform generated by CPG network

\section{Walking Simulation Based on ADAMS}

Importing the BRHL prototype model established in SolidWorks into ADAMS, the parts which don't move relatively and have same material are integrated by boolean operation. The constraints and the gait which is obtained by CPG are added to the BRHL prototype mode. Then setting the simulation time 1.2s one cycle, the gait simulation result is shown in Figure 7.
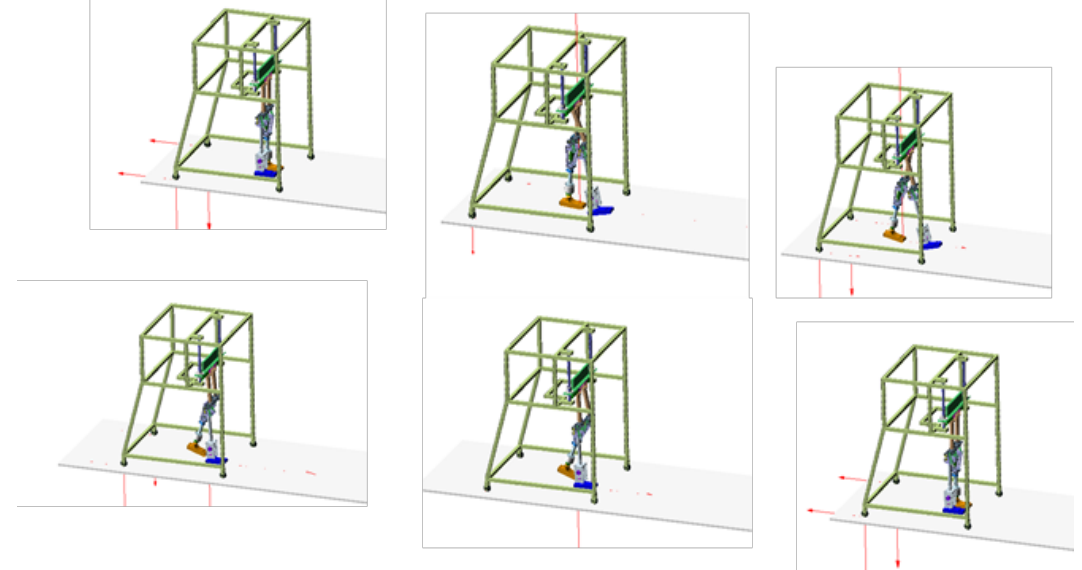

Fig.7. Gait simulation on ADAMS 
It can be seen from the above chart that the BRHL prototype model can walk naturally, which indicates that the gait planning of artificial leg by CPG is quite reasonable.

\section{Conclusion}

To achieve the artificial leg of BRHL humanoid walking, the gait planning of artificial leg was researched in this paper based on CPG. In view of the fact that hopf nonlinear oscillator can produce a stable and anti-interference periodic oscillation signal, in this paper, the CPG network was built with 6 hopf nonlinear oscillators for BRHL. The parameters of CPG network were optimized by genetic algorithm, which satisfies the needs of maximum stability and minimum energy consumption of the artificial leg gait. The walking simulation of BRHL was carried out based on ADAMS, the walking simulation result shows that the gait of the artificial leg was consistent with the human gait and the rationality of the gait planning of artificial leg by CPG network was verified.

\section{Acknowledgement}

This work was supported by the National Natural Science Foundation of China (Grant No.51105070, 51505072), Basic Research Project of Key Laboratory of Education Department of Liaoning Province (Grant No. LZ2015037) and the Fundamental Research Funds for the Central Universities of the Ministry of Education of China (Grant No. N140305001). We are sincerely grateful for the support.

\section{References}

[1] HL Xie, ZB Liu, JY Yang. Modelling of magnetorheological damper for intelligent bionic leg and simulation of knee joint movement control [J]. INT J SIMUL MODEL, 2016, 15(1):144-156.

[2] Grillner S, Wallen P. Central pattern generators for locomotion, with special reference to vertebrates [J]. Annual Review of Neuroscience, 1985, 8(6): 233-261.

[3] Sandro Nicole, Eliano Pessa. A network model with auto-oscillating output and dynamic conections [J]. Biological Cybernetics, 1993, 70(3): 275-280.

[4] Holk Cruse, Thomas Kindermann, Michael Schumm. Walknet-a biologically inspired network to control six-legged walking [J]. Neural Networks, 1998, 11(7-8): 1435-1447.

[5] Fukuoka Y, Kimura H and so on. Adaptive dynamic walking of a quadruped robot 'Tekken' on irregular terrain using a neural system model [A]. IEEE International Conference on Robotics \& Automation [C], 2003, 2: 2037-2042.

[6] Kimura H, Fukuoka Y. Adaptive dynamic walking of a quadruped robot on irregular terrain by using neural system model [A]. IEEE/RSJ International Conference on Intelligent Robots \& Systems [C], 2000, 2: 979-984.

[7] Long Wang, Shuo Wang and so on. Motion control of a robot fish based on CPG [A]. IEEE International Conference on Industrial Technology [C], 2006:1263-1268.

[8] Q Lu, J Tian. Research on walking gait of biped robot based on a modified CPG model [J]. Mathematical Problems in Engineering, 2015, 2015:1-9.

[9] A Hojjat, KC Sarma. Fuzzy genetic algorithm for optimization of steel structures [J]. Journal of Structural Engineering, 2014, 126(5):596-604.

[10] VDR Looij, HH Nijmeijer, DD Kostic. Feedback control of zero moment point for stable bipedal walking [J]. University of Hull, 2014, 32(2):161-175. 